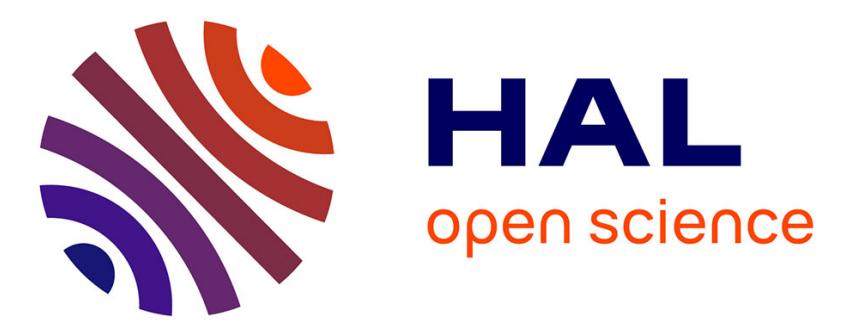

\title{
Unifying femtosecond and picosecond single-pulse magnetic switching in Gd-Fe-Co
}

F Jakobs, T A Ostler, C-H Lambert, Y Yang, S Salahuddin, R B Wilson, J. Gorchon, J Bokor, U Atxitia

\section{- To cite this version:}

F Jakobs, T A Ostler, C-H Lambert, Y Yang, S Salahuddin, et al.. Unifying femtosecond and picosecond single-pulse magnetic switching in Gd-Fe-Co. Physical Review B, 2021, 103, 10.1103/physrevb.103.104422 . hal-03377129

\section{HAL Id: hal-03377129 \\ https://hal.science/hal-03377129}

Submitted on 14 Oct 2021

HAL is a multi-disciplinary open access archive for the deposit and dissemination of scientific research documents, whether they are published or not. The documents may come from teaching and research institutions in France or abroad, or from public or private research centers.
L'archive ouverte pluridisciplinaire HAL, est destinée au dépôt et à la diffusion de documents scientifiques de niveau recherche, publiés ou non, émanant des établissements d'enseignement et de recherche français ou étrangers, des laboratoires publics ou privés. 


\title{
Unifying femtosecond and picosecond single-pulse magnetic switching in Gd-Fe-Co
}

\author{
F. Jakobs, ${ }^{1}$ T. A. Ostler,${ }^{2,3}$ C.-H. Lambert,,${ }^{4}{ }^{*}$ Y. Yang,,${ }^{5}{ }^{\dagger}$ S. Salahuddin, ${ }^{4,6}$ R. B. Wilson, ${ }^{7}$ \\ J. Gorchon $\odot, 4,6, \$$ J. Bokor $\odot,{ }^{4,6}$ and U. Atxitia $\oplus^{1, \S}$ \\ ${ }^{1}$ Dahlem Center for Complex Quantum Systems and Fachbereich Physik, Freie Universität Berlin, 14195 Berlin, Germany \\ ${ }^{2}$ Département de Physique, Universitè de Liége (B5), B-4000 Liége, Belgium \\ ${ }^{3}$ College of Business, Technology and Engineering, Sheffield Hallam University, Howard Street, Sheffield, S1 1WB, United Kingdom \\ ${ }^{4}$ Department of Electrical Engineering and Computer Sciences, University of California, Berkeley, California 94720, USA \\ ${ }^{5}$ Department of Materials Science and Engineering, University of California, Berkeley, California 94720, USA \\ ${ }^{6}$ Lawrence Berkeley National Laboratory, 1 Cyclotron Road, Berkeley, California 94720, USA \\ ${ }^{7}$ Department of Mechanical Engineering and Materials Science and Engineering Program, \\ University of California, Riverside, California 92521, USA
}

(Received 20 May 2020; revised 23 September 2020; accepted 8 February 2021; published 15 March 2021)

\begin{abstract}
Many questions are still open regarding the physical mechanisms behind the magnetic switching in $\mathrm{Gd}-\mathrm{Fe}-\mathrm{Co}$ alloys by single optical pulses. Phenomenological models suggest a femtosecond scale exchange relaxation between sublattice magnetization as the driving mechanism for switching. The recent observation of thermally induced switching in Gd-Fe-Co by using both several picosecond optical laser pulse as well as electric current pulses has questioned this previous understanding. This has raised the question of whether or not the same switching mechanics are acting at the femtosecond and picosecond scales. In this work, we aim at filling this gap in the understanding of the switching mechanisms behind thermal single-pulse switching. To that end, we have studied experimentally thermal single-pulse switching in Gd-Fe-Co alloys, for a wide range of system parameters, such as composition, laser power, and pulse duration. We provide a quantitative description of the switching dynamics using atomistic spin dynamics methods with excellent agreement between the model and our experiments across a wide range of parameters and timescales, ranging from femtoseconds to picoseconds. Furthermore, we find distinct element-specific damping parameters as a key ingredient for switching with long picosecond pulses and argue that switching with pulse durations as long as $15 \mathrm{ps}$ is possible due to a low damping constant of Gd. Our findings can be easily extended to speed up dynamics in other contexts where ferrimagnetic Gd-Fe-Co alloys have been already demonstrated to show fast and energy-efficient processes, e.g., domain-wall motion in a track and spin-orbit torque switching in spintronics devices.
\end{abstract}

DOI: 10.1103/PhysRevB.103.104422

\section{INTRODUCTION}

The speed of switching between two stable magnetic states has become a major bottleneck for future advancement of magnetic-based information technologies. The use of femtosecond laser pulses emerged as a promising solution for the ultrafast control of magnetism by the demonstration of subpicosecond spin dynamics in nickel [1,2]. The number of potential applications of this ultrafast spin dynamics quickly increased after the discovery of single-pulse switching of the magnetic polarity in ferrimagnetic Gd-Fe-Co alloys [3-6]. Further development of devices based on singlepulse switching, e.g., magnetic tunnel junctions, needs not only a complete understanding of the switching mechanisms,

*Present address: ETH Zurich, Hönggerbergring 64, 8093 Zürich, Switzerland.

${ }^{\dagger}$ Present address: Portland Technology Development Department, Intel Corp. Hillsboro, OR, 97006, USA.

${ }^{\ddagger}$ Present address: Universite de Lorraine, CNRS, IJL, F-54000

Nancy, France; jon.gorchon@univ-lorraine.fr

${ }^{\S}$ Corresponding author: unai.atxitia@fu-berlin.de but also providing computational means for realistic design of, for example, spintronics operations using ferrimagnets, such as the energy-efficient spin-orbit switching [7-9] and highvelocity domain-wall motion driven by fields [10], electric currents [11], and thermal gradients [12].

It was shown that the heat provided by the femtosecond optical pulse alone is already a sufficient stimulus to switch the magnetization $[5,13,14]$. Since the commonly accepted driving mechanism is based on faster exchange of angular momentum between sublattices ( $\sim 100 \mathrm{fs})$ than magnetization relaxation to the medium, the efficiency of such a mechanism should be drastically reduced at longer timescales [14]. This picture was contested by the observation of both thermal single-pulse all-optical switching (AOS) in Gd-Fe-Co alloys using laser pulse durations ranging from 50 fs up to 15 ps $[15,16]$ and by the heat produced by picosecond electric pulses [17]. Despite intense research to establish a robust theoretical framework for the quantitative description of thermal single-pulse (optical or electrical origin) AOS in Gd-Fe-Co, a unified picture is missing [14,18-22]. It is furthermore unclear whether the proven theoretical models for fs-pulse switching are able to describe the (up to two orders of magnitude larger) picosecond scale pulse switching. Recent experimental and 
theoretical work using phenomenological models suggested distinguished different relaxation pathways for femtosecond and picosecond pulses [23].

One of the most promising techniques for achieving a unified picture are atomistic spin dynamics (ASD) methods. They have demonstrated the ability to adequately describe the equilibrium properties of Gd-Fe-Co alloys [24] and to describe the nonequilibrium dynamics upon femtosecond laser excitation qualitatively, such as a transient ferromagneticlike state [4], thermal single-pulse AOS [5], rapid magnon localization and coalescence [25]. Furthermore, ASD methods have provided a range of predictions about the behaviors of the switching as a function of $\mathrm{Gd}$ concentration, ambient (or initial) temperature, and laser fluence $[26,27]$.

In this work we provide a unified picture of the singlepulse all-optical switching induced by pulses with durations spanning three orders of magnitude, from femtoseconds to picoseconds. To do so, we use atomistic spin dynamics methods and pump-probe experiments of single-pulse all-optical switching in Gd-Fe-Co alloys. We show that quantitative agreement between theory and experiment is achieved when element-specific damping parameters are considered in the model. These combined studies allow us to uncover the underlying physics behind magnetic switching using heat pulses up to several picoseconds in duration. Further, based on our model, we find optimal conditions for laser and material to allow switching of pulse durations up to $15 \mathrm{ps}$.

\section{EXPERIMENTAL SETUP AND MODEL}

\section{A. Experimental setup}

The experiments were carried out on a series of $\mathrm{Gd}_{x}\left(\mathrm{Fe}_{90} \mathrm{Co}_{10}\right)_{100-x}$ films of concentrations from $x=24 \%$ to $32 \%$ grown by cosputtering of the following stacks (in $\mathrm{nm}$ ): $\mathrm{Si} / \mathrm{SiO}_{2}(100) / \mathrm{Ta}(5) / \mathrm{Gd}-\mathrm{Fe}-\mathrm{Co}(20) / \mathrm{Pt}(5)$. The sample is not crystallized and instead has an amorphous structure. Hysteresis loops were measured using magneto-optic Kerr effect (MOKE) at room temperature [Fig. 1(a)]. All samples exhibited perpendicular magnetic anisotropy, and the coercivity $H_{c}$ is extracted from the hysteresis loops [Fig. 1(a)]. The coercive field $H_{c}$ increases and the polarity of the hysteresis loops reverses in sign at concentration values of around $x=28 \%$ and $29 \% \mathrm{Gd}$, which indicates the existence of a magnetization compensation point at those concentrations at $300 \mathrm{~K}$ [Fig. 1(b)]. An amplified 250-kHz Ti:sapphire laser with 810 -nm center wavelength was used for generating the highenergy pulses and as a time-resolved probe (Coherent RegA). The laser pulse duration full width at half-maximum (FWHM) was tunable from $\Delta t=55$ fs to $\Delta t=15$ ps by adjusting the final pulse compressor in the chirped pulse amplifier. Individual single-shot laser pulses could be obtained from our laser system. A MOKE microscope was used for imaging the sample magnetization after each single-laser-pulse shot and check for the reversal at various pulse energies. The system also allows one to obtain time-resolved MOKE data in a pump-probe fashion. However, when stretching the pulse duration for the pump, the probe stretches equally, reducing the experimental time resolution. The probe was focused through a $50 \times$ objective down to a size of about $1-2 \mu \mathrm{m}$. The pump

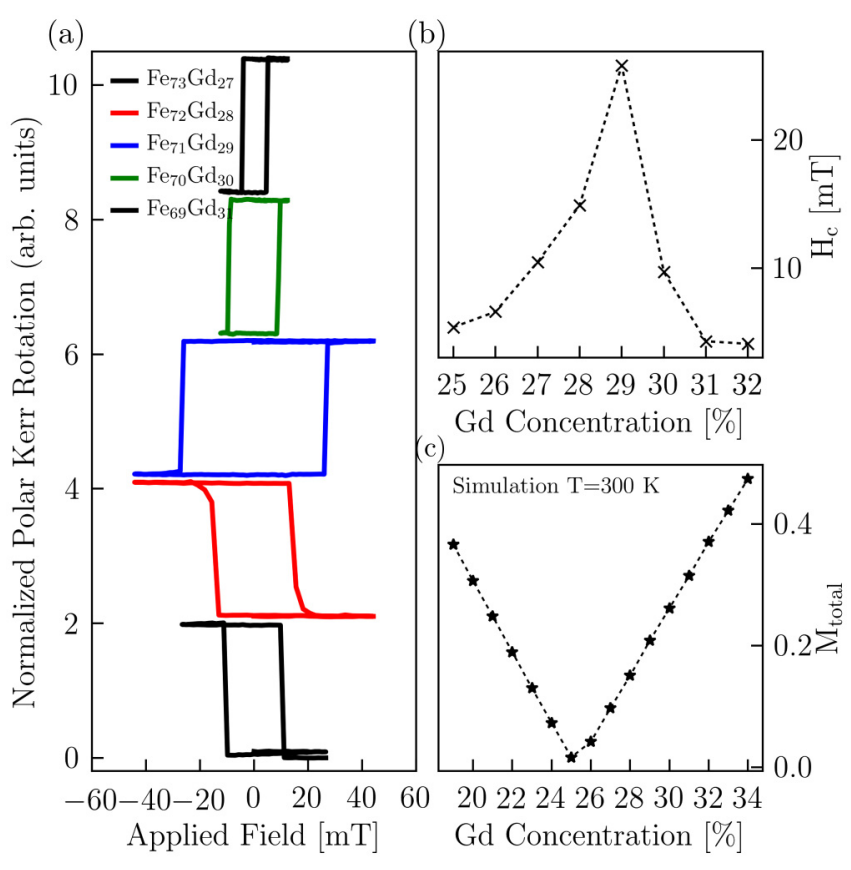

FIG. 1. (a) Magnetic hysteresis of different Fe-Gd alloys between $27 \%-31 \%$ gadolinium probed by the magneto-optical Kerr rotation at $300 \mathrm{~K}$. The use of an objective lens that is close to the electromagnet's gap induces an additional slope on the Kerr hysteresis data, due to a Faraday effect in the lenses' glass. This slope, which is independent of the sample, was numerically removed from this figure. The polarity of the hysteresis changes as the system crosses the compensation. The coercivity diverges as the Gd concentration approaches the compensation point. (b) The experimentally measured coercive field $H_{c}$ of (a) as function of the Gd concentration. The line is guidance to the eye. (c) The simulated total magnetization of the Fe-Gd alloy at $300 \mathrm{~K}$ as function of the Gd concentration. The simulated magnetization compensation temperature is slightly lower (between 25\%-26\%) than the experimental one with the drawn line being guidance to the eye.

was focused via a 15-cm lens. We note that pump-probe experiments demonstrating switching dynamics require an external applied out-of-plane magnetic field of around $10 \mathrm{mT}$ in order to reset the magnetization after each pulse event. Details of the pump-probe and microscope setup can be found in Ref. [28].

\section{B. Model}

We use an atomistic spin model based on the classical Heisenberg spin Hamiltonian

$$
\mathcal{H}=-\sum_{i \neq j} J_{i j} \mathbf{S}_{i} \cdot \mathbf{S}_{j}-\sum_{i} d_{z} S_{z}^{2}
$$

$\mathbf{S}_{i}=\boldsymbol{\mu}_{i} / \mu_{s, i}$ represents a classical, normalized spin vector at site $i$ with $\mu_{s, i}$ being the atomic magnetic moment of each sublattice. The spin at site $i, \mathbf{S}_{i}$, couples to the neighboring spin, $\mathbf{S}_{j}$ via the coupling constant $J_{i j}$. The second term of the Hamiltonian describes the onsite anisotropy with easy axis along the $z$ axis with constant anisotropy energy $d_{z}$. The lattice structure of Gd-Fe-Co is amorphous and thus difficult to fully characterize [4]. Similar to previous works, we model $\mathrm{Gd}-\mathrm{Fe}-\mathrm{Co}$ alloys as a two-sublattice system with Fe-Co being 
represented by a generic transition metal (TM) sublattice and Gd as a second sublattice that is randomly scattered throughout the TM. The simulation of $\mathrm{Fe}-\mathrm{Co}$ as one sublattice is justified by the parallel alignment of $\mathrm{Fe}$ and $\mathrm{Co}$ up to the Curie temperature and the delocalized nature of their spins. The spin dynamics is described by the atomistic stochasticLandau-Lifshitz-Gilbert equation (sLLG) [29]

$$
\frac{\left(1+\alpha_{i}^{2}\right) \mu_{s, i}}{\gamma} \frac{\partial \mathbf{S}_{i}}{\partial t}=-\left(\mathbf{S}_{i} \times \mathbf{H}_{i}\right)-\alpha_{i}\left[\mathbf{S}_{i} \times\left(\mathbf{S}_{i} \times \mathbf{H}_{i}\right)\right],
$$

where $\gamma$ is the gyromagnetic ratio and $\mu_{s, i}$ represents the magnetic moment of sublattice $i$. The phenomenological, material-dependent parameter $\alpha_{i}$ determines the rate of transfer of energy and angular momentum in and out of the magnetic system and gives rise to a damping of the spin dynamics. The damping parameter is included phenomenologically and is strongly material dependent [29]. By including a Langevin thermostat, statistical-equilibrium and nonequilibrium thermodynamic properties can be obtained. This is achieved by adding an effective fieldlike stochastic term $\zeta_{i}$ to the effective field $\mathbf{H}_{i}=\zeta_{i}(t)-\frac{\partial \mathcal{H}}{\partial \mathbf{S}_{i}}$, with whitenoise properties [30]:

$$
\left\langle\zeta_{i}(t)\right\rangle=0 \quad \text { and } \quad\left\langle\zeta_{i}(0) \zeta_{j}(t)\right\rangle=2 \alpha_{i} k_{\mathrm{B}} T_{\mathrm{el}} \mu_{s, i} \delta_{i j} \delta(t) / \gamma .
$$

The noise represents the effect of the hot itinerant electrons onto the two sublattices of localized spins. The electron temperature $T_{\mathrm{el}}$ is therefore used to scale the noise and has an indirect impact on the spin dynamics via the stochastic field $\zeta(t)$ entering the sLLG. Throughout all simulations, no external magnetic field was applied. It is a fair approximation to neglect the applied magnetic fields in our model as the energy scale of the Zeeman interaction is much smaller than the exchange interaction which drives the demagnetization and switching processes. Only on much longer timescales does the magnetic field become important.

In our computational model, we consider a spin simple cubic lattice composed of two spin sublattices Fe and Gd with dimensions of $N=160 \times 160 \times 160 \approx 4 \times 10^{6}$ spins. This system size yields minimal boundary effects and provides a large enough number of spins for calculating and averaging macroscopic parameters. To handle the computational effort of solving the sLLG for over $4 \times 10^{6}$ spins, the simulations were performed on GPUs making use of the Nvidia CUDA C-API [31].

We use the so-called two-temperature model (TTM) to describe the temporal changes in the electron and phonon temperature $\left(T_{\mathrm{ph}}\right)[32,33]$ :

$$
\begin{gathered}
C_{\mathrm{el}} \frac{\partial T_{\mathrm{el}}}{\partial t}=-g_{\mathrm{ep}}\left(T_{\mathrm{el}}-T_{\mathrm{ph}}\right)+P_{l}(t), \\
C_{\mathrm{ph}} \frac{\partial T_{\mathrm{ph}}}{\partial t}=+g_{\mathrm{ep}}\left(T_{\mathrm{el}}-T_{\mathrm{ph}}\right) .
\end{gathered}
$$

$C_{\mathrm{el}}$ and $C_{\mathrm{ph}}$ represent the specific heat of the electron and phonon system. Here, $P_{l}(t)$ represents the absorbed energy by the electron system, coming from the laser. All of the material parameters used in this study are listed in Table I and are taken from Ref. [27].

Figure 2(b) shows an example of the resulting $T_{\mathrm{el}}$ and $T_{\mathrm{ph}}$ dynamics upon application of a $100-\mathrm{fs}$ laser pulse. Due
TABLE I. Table of the Heisenberg spin Hamiltonian parameters (left) and the two-temperature model (TTM) (right). Values are taken from Ref. [27].

\begin{tabular}{lcccccc}
\hline \hline $\mathcal{H}$ & Value & & Units & TTM & & Units \\
\hline$J_{\mathrm{Fe}-\mathrm{Fe}}$ & 3.46 & $\times 10^{-21}$ & $(\mathrm{~J})$ & $\mathrm{C}_{\mathrm{ph}}$ & $3 \times 10^{6}$ & $\left(\mathrm{~J} / \mathrm{Km}^{3}\right)$ \\
$J_{\mathrm{Gd}-\mathrm{Gd}}$ & 1.389 & $\times 10^{-21}$ & $(\mathrm{~J})$ & $\mathrm{C}_{\mathrm{el}}$ & $\gamma_{\mathrm{el}} T_{\mathrm{e}}$ & \\
$J_{\mathrm{Fe}-\mathrm{Gd}}$ & -1.205 & $\times 10^{-21}$ & $(\mathrm{~J})$ & $\gamma_{\mathrm{el}}$ & 700 & $\left(\mathrm{~J} / \mathrm{Km}^{3}\right)$ \\
$\gamma_{\mathrm{Fe} / \mathrm{Gd}}$ & 1.76 & $\times 10^{-21}$ & $\left(\frac{1}{\mathrm{Ts}}\right)$ & $\mathrm{g}_{\mathrm{ep}}$ & $6 \times 10^{17}$ & $\left(\mathrm{~J} / \mathrm{sKm}^{3}\right)$ \\
$d_{z}$ & 8.072 & $\times 10^{-22}$ & $(\mathrm{~J})$ & & & \\
$\mu_{\mathrm{s}, \mathrm{Fe}}$ & 1.92 & & $\left(\mu_{\mathrm{B}}\right)$ & & & \\
$\mu_{\mathrm{s}, \mathrm{Gd}}$ & 7.63 & & $\left(\mu_{\mathrm{B}}\right)$ & & & \\
$\alpha_{\mathrm{Gd}}$ & 0.01 & & & & & \\
$\alpha_{\mathrm{Fe}}$ & Varied & & & & & \\
\hline \hline
\end{tabular}

to the low heat capacity of the electrons, the $T_{\mathrm{el}}$ increases within the same timescale of the laser pulse (shaded area) and can reach up to several thousand Kelvin. When $T_{\mathrm{el}}$ and $T_{\mathrm{ph}}$ are out of equilibrium, the electron-phonon coupling drives a transfer of energy from the electrons to the phonons, cooling the hot electron system and heating the lattice within a couple of picoseconds. As the pulse duration increases, the situation slowly changes until the timescales of the laser excitation and electron-phonon relaxation processes become similar. Figure 2(a) shows, as an example, the $T_{\mathrm{el}}$ and $T_{\mathrm{ph}}$ dynamics for a laser pulse duration of $1 \mathrm{ps}$. In this case, the energy transfer from the electrons and phonons acts on almost the same timescale as the energy load from the laser to the electrons. The direct consequence is that for the same absorbed energy, the maximum temperature reached by the electron system is reduced as the pulse duration increases. Ultimately, for very long pulses the dynamics of the electron and phonon temperature becomes the same and the steep $T_{\mathrm{el}}$ increase disappears.

Figure 3 shows the corresponding magnetic response of the $\mathrm{Fe}$ and the Gd sublattices to the discussed 1-ps pulse for different fluences [Figs. 3(a)-3(c)]. We note that in the simulations a new equilibrium state is reached fairly quickly after about 7-8 ps after the initial excitation. At that point, $T_{\mathrm{el}}$ and $T_{\mathrm{ph}}$ are equilibrated and both sublattices have adjusted to the new electron temperature. Additional effects that slow down the reordering process, such as domain formation or heat transport, are not considered.

\section{QUANTITATIVE COMPARISON BETWEEN EXPERIMENTS AND SIMULATIONS}

Figure 2(c) shows a direct, quantitative description of the dynamics of thermal single-pulse magnetic switching of Gd-Fe-Co alloys using femtosecond- and picosecond-pulse durations. The figure depicts the $z$ component of the normalized magnetization $m$ of the Fe sublattice for a pulse duration of 1 ps (left) and 100 fs (right) with experimental measurements being shown as green points and computer simulations in red. ${ }^{1}$ The laser fluence used is sufficient to

\footnotetext{
${ }^{1}$ In our setup it is not possible to measure the pulse duration after the objective (because we need a collimated beam for the autocorre-
} 
(a)

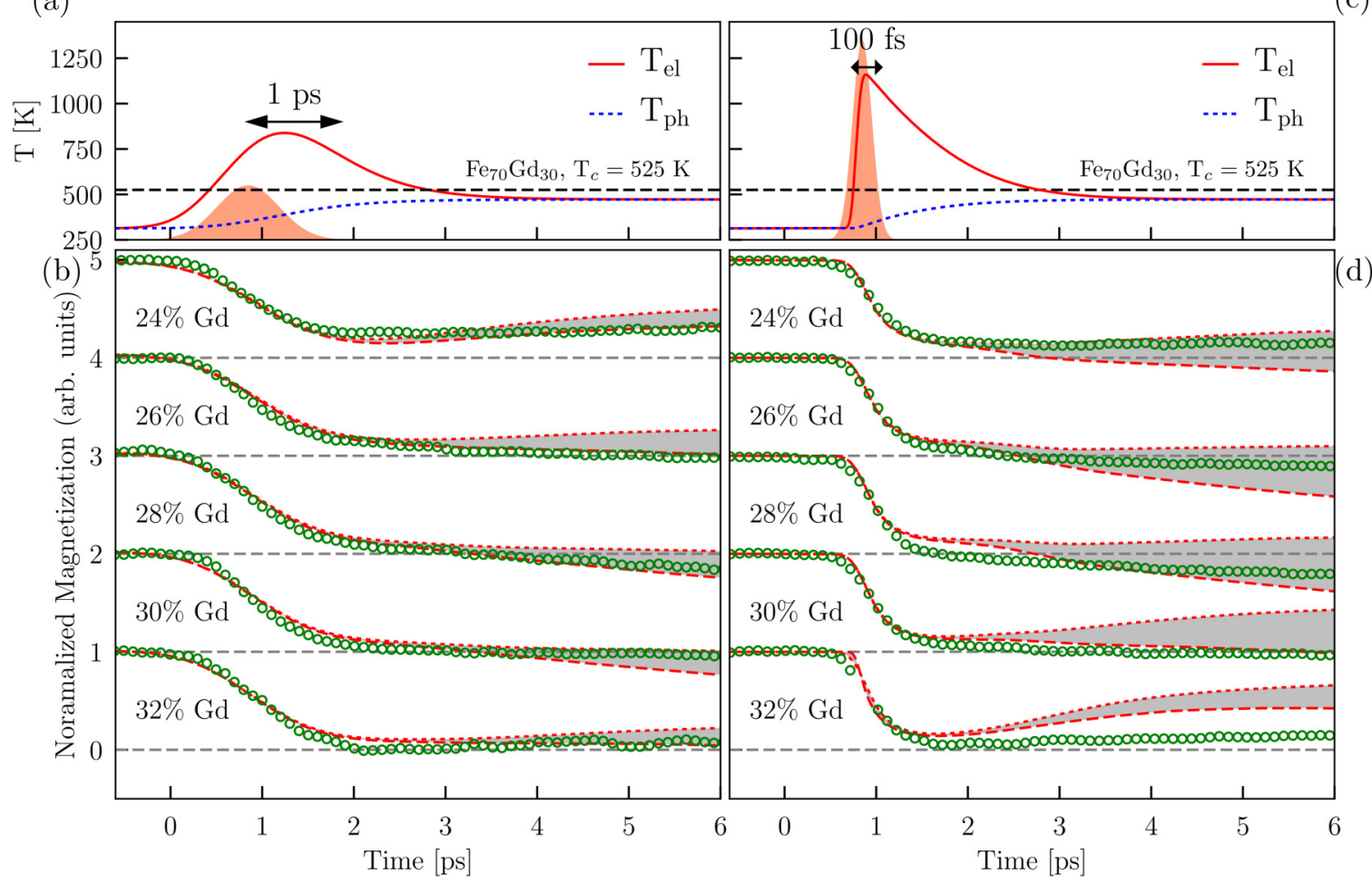

FIG. 2. The temperature dynamics of the $T_{\mathrm{el}}$ (solid red) and $T_{\mathrm{ph}}$ (blue dotted) for a pulse duration of (a) $1 \mathrm{ps}$ and (b) $100 \mathrm{fs}$. The total energy of the pulse is the same for both pulse durations. (c), (d) Atomistic spin dynamics simulations (red dotted lines) and experimental measurements (large green dots) of the magnetization dynamics of the Fe sublattice for a range of Gd concentrations at $300 \mathrm{~K}$. The experimental normalized magnetization is obtained through a shifting and normalization of the measured pump-induced change in magnetization $\left(M_{s}+\Delta M_{z}\right) / M_{s}$, where $M_{s}$ is the saturation magnetization. The simulated normalized magnetization is obtained by dividing the averaged magnetic moment by the average magnetization at $300 \mathrm{~K}$ of each sublattice. The plots for each Gd concentration are shifted by an offset of 1 among each other. The simulations correspond to a Gaussian weighted average of multiple simulations of different Gd concentrations with a variance of $\sigma^{2}=5.76 \%$. The gray area between the dotted red lines indicates a variation in the laser fluence of $\approx \pm 0.5 \%$ of a chosen mean fluence. Due to the overlapping of pump and probe pulse in the experiment and for direct comparison, the dynamics coming out from the simulations is convoluted with a 800 -fs probe pulse, and the simulations for the 100 -fs pulse are convoluted using a 250 -fs probe pulse.

achieve AOS for the Gd concentrations between $26 \%$ and $30 \%$ [Figs. 2(c) and 2(d)] for both pulse durations. We estimate the absorbed fluence to be around $1.5 \mathrm{~mJ} / \mathrm{cm}^{2}$ (assuming a $30 \%$ absorption [16]), which corresponds to about $5 \times 10^{8} \mathrm{~J} / \mathrm{m}^{3}$ for our 30-nm-thick metallic film. We note that an important distinction between the experiments' and simulations' energy absorption scenarios is that in the experiments we find an exponentially decaying absorption profile, whereas in the simulations the full magnetic layer can be set to absorb a homogeneous energy density. To account for potential fluctuations of the laser fluence during data acquisition, two different results from simulations for laser fluences with a variation of $0.5 \%$ are shown as red dotted and dashed lines in Figs. 2(c) and 2(d). Importantly, laser and material parameters

lator), so we do not have a measure of the probe pulse duration after the objective. We estimate the pulse to stretch by about $160 \mathrm{fs}$ to $240 \mathrm{fs}$ after the objective based on the 50-nm bandwidth of the laser and assuming 3 to $4.5 \mathrm{~cm}$ of UV fused silica glass for the optics. Based on this we estimate the probe pulse to $100 \mathrm{fs}+200 \mathrm{fs}=$ $300 \mathrm{fs}$ and $1 \mathrm{ps}-200 \mathrm{fs}=800 \mathrm{fs}$ in the two experimental conditions of Fig. 2. in this section were kept constant throughout all simulations. The intrinsic damping parameters $\alpha_{\mathrm{Fe}}$ and $\alpha_{\mathrm{Gd}}$ for the $\mathrm{Fe}$ and Gd sublattices were set to $\alpha_{\mathrm{Fe}}=0.06$ and $\alpha_{\mathrm{Gd}}=0.01$. The inclusion of the element-specific nature of the damping in our model is one of the key factors that allowed us to quantitatively describe our experimental measurements. In a recent work on single-pulse AOS in Tb-Gd-Fe-Co alloys, similar conclusions have been drawn about the role of distinct damping parameters in AOS [34]. These damping parameters are in agreement with the ultrafast spin dynamics measured in the respective pure materials $[35,36]$. While $\mathrm{Fe}$ and $\mathrm{Co}$ demagnetize on timescales of hundreds of femtoseconds, the rare-earth $\mathrm{Gd}$ responds much slower to optical excitation [4]. It has been argued that the reason behind these slow dynamics is the localized character of the $4 f$ spins and the absence of orbital angular momentum [37]. In previous works, the same damping value is consistently used for both sublattices. However, the good quantitative agreement between our experiments and the model suggests that the damping parameter for rare-earth (RE) metals used in sLLG models should be lower than the one used in transition metals. This picture of larger damping for Fe-Co than for Gd aligns with recent observations on magnetic domain-wall mobility in $\mathrm{Gd}-\mathrm{Fe}$ - 


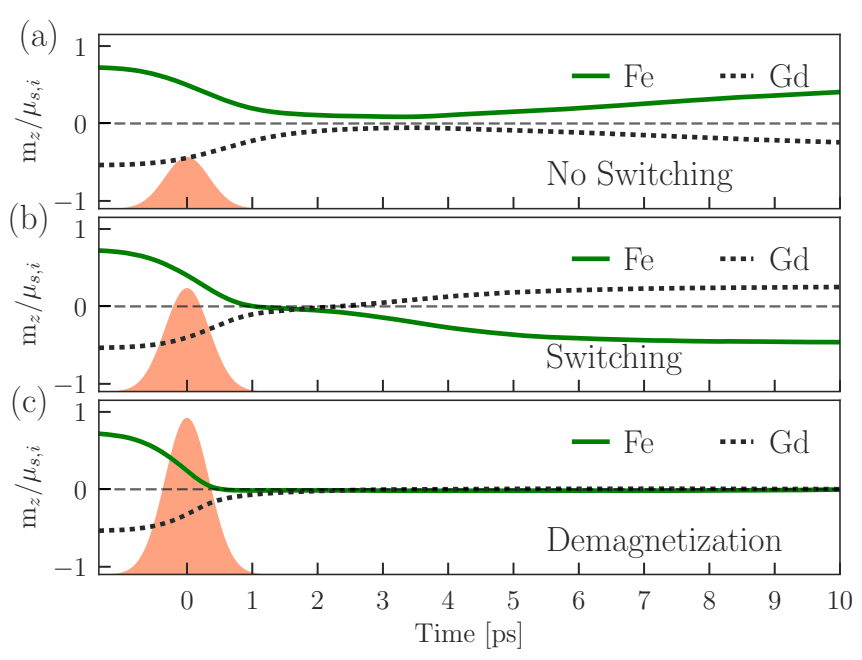

FIG. 3. The (normalized) magnetization dynamics for Fe (green) and Gd (black) after excitation by different fluences of a 1-ps laser pulse. (a) The magnetization dynamics for a weak 1-ps pulse that does not switch the alloy. (b) The dynamics for an increased fluence that does switch Gd-Fe. (c) The dynamics for a high fluence that demagnetizes the alloy. The dynamics of the two-temperature model for a similar laser excitation is shown in Fig. 2(a).

Co [38]. Our choice of larger damping value for Fe-Co than for $\mathrm{Gd}$ is in agreement with those observations. We note that since the laser probes areas of tens of micrometers, it is important to consider the chemically inhomogeneous nature of the experimental samples with locally varied Gd concentrations [39]. The switching behavior within these chemical inhomogeneities strongly depends on local system parameters, especially the Curie temperature $T_{c}$, which varies with the $\mathrm{Gd}$ concentration. For example, a $\mathrm{Fe}_{75} \mathrm{Gd}_{25}$ alloy shows a $T_{c} \approx 560 \mathrm{~K}$ while a $\mathrm{Fe}_{66} \mathrm{Gd}_{34}$ alloy only has a $T_{c} \approx 500 \mathrm{~K}$. The influence of such chemical inhomogeneities is especially relevant when working close to the critical laser fluence, which marks the energy threshold for switching and nonswitching behavior. Close to this fluence level one region with a Gd concentration might switch for a given fluence while another Gd concentration does not switch for the same fluence (see Fig. 3). Therefore, we take a weighted (Gaussian) average of independent simulations of different Gd concentrations with a variance of $\sigma^{2}=5.76 \% \mathrm{Gd}$, which yielded the best agreement with our experiments. The expectation value $\mu$ of the distribution was set to the experimentally indicated one ( $\mu=x$ for an $\mathrm{Fe}_{1-x} \mathrm{Gd}_{x}$ alloy). The actual distribution variance in our experiments is unknown, however, we explored values around the experimentally measured ones by Graves and coworkers [39]. This agreement is robust, varying $\sigma$ by $10 \%-20 \%$ yielded similarly good agreement. As discussed in the previous section and shown in Figs. 2(c) and 2(d), the magnetization dynamics in the simulations reaches an equilibrium state after a couple of picoseconds. Additional effects, relevant on longer timescales such as domain formation, heat transport, or small applied magnetic fields used in the experiments are not considered in this model. Therefore, a comparison to experimental results on timescales of several hundred picoseconds is beyond the scope of this work.
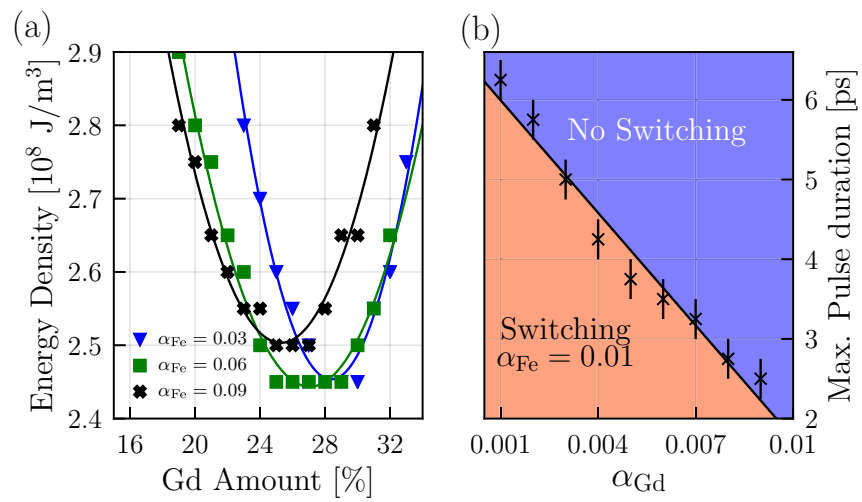

FIG. 4. Simulation results for (a) critical laser fluence of a 350-fs pulse as function of the $\mathrm{Gd}$ concentration $\left(\alpha_{\mathrm{Gd}}=0.01\right.$ constant) for $\alpha_{\mathrm{Fe}}=0.03$ (blue triangles), 0.06 (green boxes), 0.09 (black crosses). The lines represent an $x^{2}$ fitting and serve only as a guide to the eye. (b) Maximum pulse duration achieved in the model as function of the Gd damping $\alpha_{\mathrm{Gd}}\left(\alpha_{\mathrm{Fe}}=0.01\right.$ constant) for an $\mathrm{Fe}_{75} \mathrm{Gd}_{25}$ alloy.

To conclude this section we found that atomistic spin models are sufficient for a quantitative description of our experiments for a wide range of pulse durations and Gd concentrations with only a single set of parameters for all of them.

\section{OPTIMAL CONDITIONS FOR PICOSECOND PULSE SWITCHING}

In this section we investigate the robustness of our findings and explore the ideal material and laser conditions necessary for energy-efficient switching in $\mathrm{Gd}-\mathrm{Fe}-\mathrm{Co}$. Previous models have suggested that a distinct demagnetization time $\tau$ is necessary to achieve switching. The damping $\alpha_{i}$ at site $i$ is one of the key parameters for controlling $\tau_{i}$ as previous works in ferrimagnets suggest a $\tau_{i} \propto \mu_{i} / \alpha_{i}$ scaling [40]. Based on the same arguments, one could imagine that the maximum pulse duration within the used model also depends on the intrinsic demagnetization timescales. Indeed, a detailed understanding about the role of damping parameters on switching efficiency could be used to tailor optimized dissipative paths in engineered heterostructures. Thus, in the following we study the dependence of the critical fluence and the maximal pulse duration on the intrinsic damping.

In the previous section we used $\alpha_{\mathrm{Fe}}=0.06$ and $\alpha_{\mathrm{Gd}}=$ 0.01 . However, these values are of phenomenological origin, chosen to match our experiments. In the following, we explore switching behavior for damping values of higher and lower $\alpha_{\mathrm{Fe}}$ while keeping $\alpha_{\mathrm{Gd}}$ constant. Furthermore, the data gained from computer simulations that we show in the following correspond to a fixed Gd concentration. In comparison, when we compare the results of the model and those of the experiment, we accounted for the chemical inhomogeneity of the samples by averaging the magnetization dynamics data over a range of concentrations. Consequently, their dynamics are not directly comparable.

Figure 4(a) shows the critical fluence found in simulations as function of the $\mathrm{Gd}$ concentration for different $\alpha_{\mathrm{Fe}}$ in the range of $\alpha_{\mathrm{Fe}}=0.03-0.09$ while keeping a fixed $\alpha_{\mathrm{Gd}}=0.01$. With an increasing damping $\alpha_{\mathrm{Fe}}$ from 0.03 to 0.09 which 


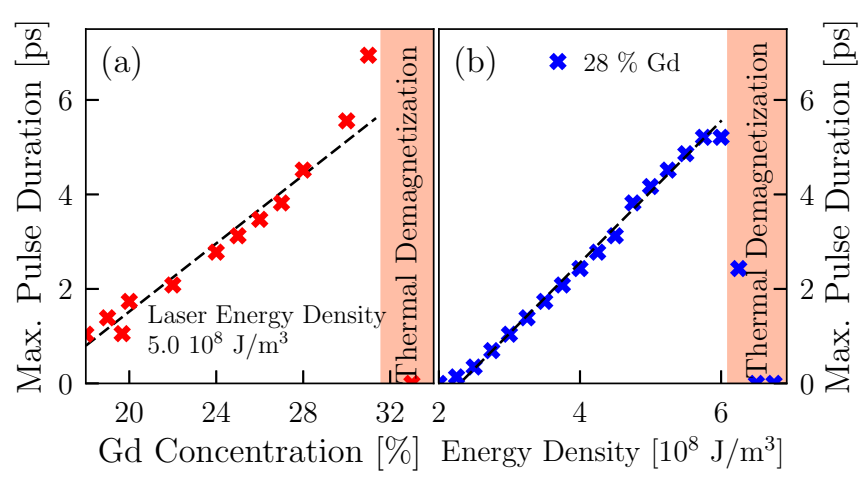

FIG. 5. (a) Maximum possible pulse duration gained from simulations as a function of the Gd concentration for a fixed laser energy. (b) Simulated maximum pulse duration as a function of the absorbed energy for a fixed $\mathrm{Gd}$ concentration of $28 \%$.

speeds up the Fe-spin dynamics, we observe a shift of the critical fluence minimum towards lower Gd concentrations from $29 \%\left(\alpha_{\mathrm{Fe}}=0.03\right)$ to $25 \%\left(\alpha_{\mathrm{Fe}}=0.09\right)$. Furthermore Fig. 4 shows a $x^{2}$ fit as a guide to the eye of the shift of the critical fluence for each Gd concentration. We observe not just a shift of the critical fluence minimum from $29 \% \mathrm{Gd}$ to $25 \%$ $\mathrm{Gd}$, but also a shift of the general switching window in the same direction.

\section{Impact of the pulse duration}

Magnetic switching driven by electric pulses is of interest for future technological applications. However, generating electrical pulses shorter than a few picoseconds is extremely difficult. Therefore, finding switching conditions to achieve single-pulse AOS with the longest possible pulses becomes a challenge. Previous experimental results estimated that laser pulses with durations of up to $10 \mathrm{ps}$ were able to switch the magnetization for a very specific $\mathrm{Gd}$ concentration, $\mathrm{Gd}_{27} \mathrm{FeCo}$ alloys [16]. For different $\mathrm{Gd}$ concentrations the maximum pulse duration within the model decreases notably, such as for $x_{\mathrm{Gd}}=24 \%$ the maximum pulse duration reduces to $1 \mathrm{ps}$ [16]. Here, we show that in order to describe single-pulse AOS, ASD simulations and the physics described by them remain valid on timescales of up to 15 ps. Figure 4(b) shows the maximum pulse duration of an $\mathrm{Fe}_{75} \mathrm{Gd}_{25}$ alloy as function of the Gd damping $\alpha_{\mathrm{Gd}}$ while keeping $\alpha_{\mathrm{Fe}}=0.01$ constant. We find a linear increase of the maximum possible pulse duration that is able to switch the alloy with a decreasing Gd damping $\alpha_{\mathrm{Gd}}$. Decreasing $\alpha_{\mathrm{Gd}}$ slows down the Gd dynamics compared to the Fe sublattice which seems to increase the maximum pulse duration. For $\alpha_{\mathrm{Fe}}=0.01$ and $\alpha_{\mathrm{Gd}}=0.001$ we were able to switch an $\mathrm{Fe}_{75} \mathrm{Gd}_{25}$ alloy in our simulations with a pulse of more than $6 \mathrm{ps}$. This is far longer than what we found in our own experiments (see Fig. 6) but is only slightly longer than the maximum pulse duration for that alloy found in Ref. [23]. Since the maximum pulse duration is highly susceptible to the ratio between dampings, $\alpha_{\mathrm{Fe}} / \alpha_{\mathrm{Gd}}$, the difference between our experiments and those in Ref. [23] could be related to a somewhat smaller damping ratio in our experiments, owning for instance to slight differences in the growing conditions. We performed further simulations with different absolute values

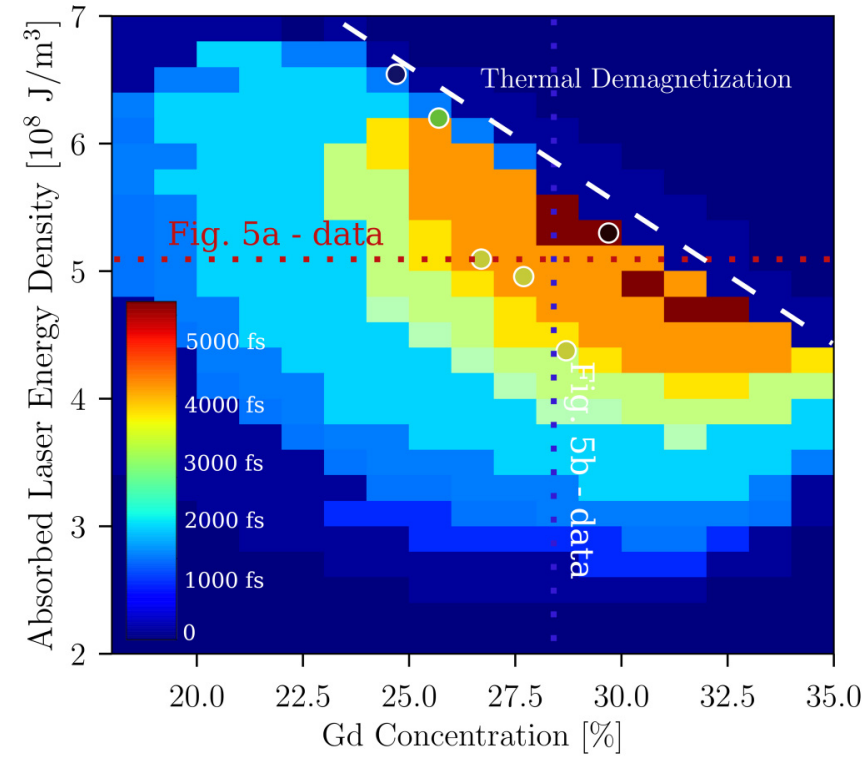

FIG. 6. Maximum laser pulse duration (as color) as function of the Gd concentration ( $x$ axis) and absorbed power density ( $y$ axis). Red color areas correspond to longer laser pulses, while blue areas only switch for short pulse durations. The damping parameters for this set of simulations were set to $\alpha_{\mathrm{Fe}}=0.03$ and $\alpha_{\mathrm{Gd}}=0.01$. For high laser fluences and high Gd concentrations the system gets completely demagnetized (top right). The experimental measurements of the maximal achievable pulse duration are shown as white circled points with the color indicating their maximum pulse duration.

of $\alpha_{\mathrm{Fe}}$ and $\alpha_{\mathrm{Gd}}$, while keeping a constant ratio $\alpha_{\mathrm{Fe}} / \alpha_{\mathrm{Gd}}$. These simulations have shown that the position of the critical fluence minimum with respect to the Gd concentration varies much with the ratio $\alpha_{\mathrm{Fe}} / \alpha_{\mathrm{Gd}}$, but only slightly with the absolute values of $\alpha_{\mathrm{Fe}}$ and $\alpha_{\mathrm{Gd}}$.

This seems to indicate that switching with ps pulses works best when the damping difference between the sublattices is as large as possible. To gain further insight into this process, we conduct computer simulations on a large set of Gd concentrations, laser fluences, and pulse durations. The goal here was to find the maximum pulse duration that switches the alloy for a given set of Gd concentrations and pulse energies. In order to do so, we first define a switching criteria: Starting from $m_{z, \mathrm{Fe}}>0$ every simulation could end up in one of the three possible states: (i) recovery $\left(m_{z, \mathrm{Fe}} \geqslant 0.12\right)$ [see Fig. 3(a)], (ii) switching $\left(m_{z, \mathrm{Fe}} \leqslant-0.12\right)$ [Fig. 3(b)], and (iii) thermal demagnetization $\left(0.12>m_{z, \mathrm{Fe}}>-0.12\right)$ [Fig. 3(c)]. The state of the system is evaluated $20 \mathrm{ps}$ after the laser excitation in order to give the spin system time to equilibrate to the final temperature. This duration should be sufficient as the system size is relatively small compared to larger domain-size features, which are important on much longer timescales. Before we present the full result as a two-dimensional (2D) color map, we first focus on two subsets of the full result.

Figure 5(a) shows the maximum possible pulse duration for a fixed total absorbed energy density of $5 \times 10^{8} \mathrm{~J} / \mathrm{m}^{3}$ that still switches the system with $\alpha_{\mathrm{Fe}}=0.03$ and $\alpha_{\mathrm{Gd}}=0.01$.

Increasing the $\mathrm{Gd}$ concentration allows for longer pulses to switch the system up to approximately $31.0 \% \mathrm{Gd}$ when the fixed total energy density of $5 \times 10^{8} \mathrm{~J} / \mathrm{m}^{3}$ causes the 
system to completely demagnetize. This is due to the decreasing Curie temperature of the sample as the $\mathrm{Gd}$ concentration increases. In Fig. 5(b) the Gd concentration is set to $28 \%$ and the total absorbed energy density is varied. In order to switch this $\mathrm{Fe}_{72} \mathrm{Gd}_{28}$ alloy with longer pulses one needs to linearly provide more energy via the laser. This is related to the electron-phonon coupling, which is already significantly acting for longer pulses while the laser pulse is still pumping energy into the electron system. This cools down the electron system temperature at a faster rate than for femtosecond laser pulses. Thus, more energy input from the laser is needed, as more energy is translated to the phonon system during the laser pulse.

Figure 6 shows the full result by combining all simulations with the color representing the maximum pulse duration as a function of the Gd concentration ( $x$ axis) and total absorbed energy density ( $y$ axis). Red colors refer to the possibility of switching the system with longer pulses (up to 6 ps for the chosen damping parameters), while areas with blue colors only allow for switching with short pulses. The top right corner with high absorbed energy densities and high Gd concentrations completely demagnetizes once a certain threshold is crossed. This area increases linearly as the Gd concentration increases, due to the linearly decreasing Curie temperature. For longer pulse durations, the allowed set of parameters that switches the Fe-Gd alloy reduces to a much narrower set (or switching window). For example, only $\mathrm{Gd}$ concentrations between $\approx 26 \% \mathrm{Gd}$ and $32 \% \mathrm{Gd}$ are able to be switched with 5-ps pulses and require a precise laser energy. Otherwise, the alloy either demagnetizes completely or recovers without switching. The experimental measurements of the maximal achievable pulse duration are shown as white circled points with the color indicating their maximum pulse duration. The overall agreement between our experiments and our model is good. However, for the $31 \%$ and the $25 \%$ Gd concentration the maximum measured pulse duration was only about $220 \mathrm{fs}$ and disagrees with the results of our model $(31 \% \mathrm{Gd}$ measurement not shown). The experimental results of Ref. [23] with psscale switching even up to $23 \%$ Gd agree quite well with our simulations. Reference [23] also finds a similar linear increase of the switching duration as the Gd concentration increases. In our analysis we used a threshold of $m_{z, \mathrm{Fe}}<-0.12$, that divides switching from demagnetization. This chosen threshold value affects the maximum pulse duration. Reducing this threshold increases the maximum pulse duration for switching. However, the shapes of the different areas in Fig. 6 are not affected by the chosen threshold value. For simplicity, in our model we neglected any heat dissipation of the Gd-Fe-Co alloy towards the substrate. The heat dissipation in the first couple of picoseconds barely affects the overall behavior of the magnetization dynamics and, consequently the switching behavior. Considering $m_{z, \mathrm{Fe}}<0$ as the switching criteria in the absence of cooling is problematic as this state can also be considered as a pure thermal demagnetized state. Further studies could include the effect of the substrate.

Furthermore, as found in the previous section, the maximum switching duration depends on the damping ratio $\alpha_{\mathrm{Fe}} / \alpha_{\mathrm{Gd}}$ [compare Fig. 4(b)]. In the simulations for Fig. 6 we used moderate values of $\alpha_{\mathrm{Fe}}=0.03$ and $\alpha_{\mathrm{Gd}}=0.01$. Using a higher ratio of $\alpha_{\mathrm{Fe}} / \alpha_{\mathrm{Gd}}$ would most likely result in longer switching durations than those seen in Fig. 6. Notably, previous experimental measurements have shown switching for pulse durations up to $15 \mathrm{ps}$ for compositions close to the magnetic compensation. Our model is also capable of reproducing such a switching duration with up to 15 ps by combining the results of this section. By selecting a high ratio between the element-specific damping parameters $\alpha_{\mathrm{Fe}}=0.01$ and $\alpha_{\mathrm{Gd}}=0.001$ and choosing optimal parameters from Fig. 6 for the pulse energy, we were able to switch a $\mathrm{Gd}_{29} \mathrm{Fe}_{71}$ alloy using a 14-ps pulse with an absorbed laser energy density of $5.95 \times 10^{8} \mathrm{~J} / \mathrm{m}^{3}$.

\section{CONCLUSIONS}

To summarize, we have conducted a joint theoretical and experimental study of single-pulse switching of various GdFe-Co alloys using a wide range of pulse durations, from a few femtoseconds up to 15 picoseconds. Our results show that switching is possible for this wide range of pulse durations of two orders of magnitude, however, the available material parameters that allow for switching reduce as the pulse duration increases. We demonstrate that the same, underlying physics utilized by atomistic spin dynamics simulations is able to describe switching within hundreds of femtoseconds, as well as tens of picoseconds.

In our experiments, the magnetization dynamics is measured using time-resolved magneto-optical Kerr measurements, which provide information on the Fe-spin sublattice dynamics. We were able to quantitatively reproduce those measurements using atomistic spin dynamics simulations (ASD) for all pulse durations used in our experiments, and a wide range of $\mathrm{Gd}$ concentrations between $24 \% \mathrm{Gd}$ up to $32 \%$. We have kept the same set of material parameters throughout all simulations, e.g., atomic magnetic moments, exchange and anisotropy constants, which demonstrates the robustness of our model. The results of this approach demonstrate that atomistic spin dynamics methods and the physics described by them in the context of single-laser-pulse all-optical switching still remain valid on timescales of up to 15 ps. One consequence of our study, based on the quantitative agreement between theory and experiment, is the necessity to consider distinct element-specific damping constants. This is in striking contrast to previous works, where only qualitative comparisons were performed. In order to achieve this quantitative agreement, we also needed to consider material inhomogeneities with respect to the Gd concentration in the model.

As for technological applications of single-pulse switching, establishing conditions for steering pulse duration able to switch magnetization in Gd-Fe-Co alloy could foster picosecond electric pulse as switching stimulus for spintronic applications. The first works utilizing picosecond electic pulses have already shown promising results [17], but quantitative computational modeling is still missing. Therefore, we believe that the insights provided by the modeling work presented here will help to better understand the conditions necessary for electric pulse switching. To explore this possibility, we have investigated computationally the optimal system parameters to achieve the longest possible pulse duration able to switch $\mathrm{Gd}-\mathrm{Fe}-\mathrm{Co}$. In agreement with recent works on single-pulse AOS in Tb-Gd-Fe-Co alloys, we found a large 
discrepancy between the distinct element-specific damping parameters to be a key parameter for longer pulse duration switching [34]. Furthermore, our results show that for long pulse durations, the set of available parameters of Gd concentrations and laser fluences, the so-called switching window, reduces continuously as the pulse duration increases. Using a well-defined, ideal set of parameters by combining various results of our work allowed us to switch a $\mathrm{Gd}_{29} \mathrm{Fe}_{71}$ alloy in an ASD simulation using a 14-ps pulse. Reference [41] showed that single-pulse AOS is not limited to alloys but it is also possible in ferrimagnetic multilayers. These kinds of heterostructures are better suited for technological applications as they allow for each layer to be tailored individually. Atomistic spin dynamics methods have been used in previous works to model ferrimagnetic multilayers and investigate the conditions for AOS using femtosecond laser pulses $[13,42,43]$. Our results provide a quantitative prediction of how composition affects single-shot switching for different material properties through varying the composition. By varying the composition, the effective exchange between antiferromagnetically coupled species is varied. One should be able to draw equivalences between this and, for example, a multilayer structure of $\mathrm{Gd}-$ Fe with different thicknesses and/or number of repeats of theses layers. However, this task goes beyond the aim of this work. Our results can furthermore help to understand AOS in other material such as the recently observed switching in $\mathrm{Mn}_{2} \mathrm{Ru}_{x} \mathrm{Ga}$ Heusler alloys [44].

\section{ACKNOWLEDGMENTS}

At the FU Berlin support by the Deutsche Forschungsgemeinschaft through SFB/TRR 227 "Ultrafast Spin Dynamics," Project A08 is gratefully acknowledged. T. A. Ostler gratefully acknowledges the support of the UK EPSRC through Grant No. EP/T027916/1 and the Vice-Chancellor's Fellowship Scheme at Sheffield Hallam University. We wish to gratefully acknowledge support by the Director, Office of Science, Office of Basic Energy Sciences, Materials Sciences and Engineering Division, of the US Department of Energy under Contract No. DE-AC02-05-CH11231, and by the National Science Foundation Center for Energy Efficient Electronics Science.
[1] E. Beaurepaire, J. C. Merle, A. Daunois, and J. Y. Bigot, Phys. Rev. Lett. 76, 4250 (1996).

[2] B. Koopmans, G. Malinowski, F. Dalla Longa, D. Steiauf, M. Fähnle, T. Roth, M. Cinchetti, and M. Aeschlimann, Nat. Mater. 9, 259 (2010).

[3] C. D. Stanciu, F. Hansteen, A. V. Kimel, A. Kirilyuk, A. Tsukamoto, A. Itoh, and T. Rasing, Phys. Rev. Lett. 99, 047601 (2007).

[4] I. Radu, K. Vahaplar, C. Stamm, T. Kachel, N. Pontius, H. A. Dürr, T. A. Ostler, J. Barker, R. F. L. Evans, R. W. Chantrell, A. Tsukamoto, A. Itoh, A. Kirilyuk, T. Rasing, and A. V. Kimel, Nature (London) 472, 205 (2011).

[5] T. A. Ostler, J. Barker, R. F. L. Evans, R. W. Chantrell, U. Atxitia, O. Chubykalo-Fesenko, S. El Moussaoui, L. Le Guyader, E. Mengotti, L. J. Heyderman, F. Nolting, A. Tsukamoto, A. Itoh, D. Afanasiev, B. A. Ivanov, A. M. Kalashnikova, K. Vahaplar, J. Mentink, A. Kirilyuk, T. Rasing, and A. V. Kimel, Nat. Commun. 3, 666 (2012).

[6] S. Mangin, M. Gottwald, C.-H. Lambert, D. Steil, V. Uhliř̌, L. Pang, M. Hehn, S. Alebrand, M. Cinchetti, G. Malinowski, Y. Fainman, M. Aeschlimann, and E. E. Fullerton, Nat. Mater. 13, 286 (2014).

[7] R. Mishra, J. Yu, X. Qiu, M. Motapothula, T. Venkatesan, and H. Yang, Phys. Rev. Lett. 118, 167201 (2017).

[8] J. Yu, D. Bang, R. Mishra, R. Ramaswamy, J. H. Oh, H. J. Park, Y. Jeong, P. Van Thach, D.-K. Lee, G. Go, S. W. Lee, Y. Wang, S. Shi, X. Qiu, H. Awano, K. J. Lee, and H. Yang, Nat. Mater. 18, 29 (2019).

[9] K. Cai, Z. Zhu, J. M. Lee, R. Mishra, L. Ren, S. D. Pollard, P. He, G. Liang, K. L. Teo, and H. Yang, Nat. Electron. 3, 37 (2020).

[10] K.-J. Kim, S. K. Kim, Y. Hirata, S.-H. Oh, T. Tono, D.-H. Kim, T. Okuno, W. S. Ham, S. Kim, G. Go, Y. Tserkovnyak, A. Tsukamoto, T. Moriyama, K.-J. Lee, and T. Ono, Nat. Mater. 16, 1187 (2017).
[11] L. Caretta, M. Mann, F. Büttner, K. Ueda, B. Pfau, C. M. Günther, P. Hessing, A. Churikova, C. Klose, M. Schneider, D. Engel, C. Marcus, D. Bono, K. Bagschik, S. Eisebitt, and G. S. D. Beach, Nat. Nanotechnol. 13, 1154 (2018).

[12] A. Donges, N. Grimm, F. Jakobs, S. Selzer, U. Ritzmann, U. Atxitia, and U. Nowak, Phys. Rev. Research 2, 013293 (2020).

[13] S. Gerlach, L. Oroszlany, D. Hinzke, S. Sievering, S. Wienholdt, L. Szunyogh, and U. Nowak, Phys. Rev. B 95, 224435 (2017).

[14] J. H. Mentink, J. Hellsvik, D. V. Afanasiev, B. A. Ivanov, A. Kirilyuk, A. V. Kimel, O. Eriksson, M. I. Katsnelson, and T. Rasing, Phys. Rev. Lett. 108, 057202 (2012).

[15] D. Steil, S. Alebrand, A. Hassdenteufel, M. Cinchetti, and M. Aeschlimann, Phys. Rev. B 84, 224408 (2011).

[16] J. Gorchon, R. B. Wilson, Y. Yang, A. Pattabi, J. Y. Chen, L. He, J. P. Wang, M. Li, and J. Bokor, Phys. Rev. B 94, 184406 (2016).

[17] Y. Yang, R. B. Wilson, J. Gorchon, C.-H. Lambert, S. Salahuddin, and J. Bokor, Sci. Adv. 3, e1603117 (2017).

[18] A. J. Schellekens and B. Koopmans, Phys. Rev. B 87, 020407(R) (2013).

[19] S. Wienholdt, D. Hinzke, K. Carva, P. M. Oppeneer, and U. Nowak, Phys. Rev. B 88, 020406(R) (2013).

[20] A. M. Kalashnikova and V. I. Kozub, Phys. Rev. B 93, 054424 (2016).

[21] V. N. Gridnev, Phys. Rev. B 98, 014427 (2018).

[22] A. V. Kimel and M. Li, Nat. Rev. Mater. 4189 (2019),.

[23] C. S. Davies, T. Janssen, J. H. Mentink, A. Tsukamoto, A. V. Kimel, A. F. G. van der Meer, A. Stupakiewicz, and A. Kirilyuk, Phys. Rev. Appl. 13, 024064 (2020).

[24] T. A. Ostler, R. F. L. Evans, R. W. Chantrell, U. Atxitia, O. Chubykalo-Fesenko, I. Radu, R. Abrudan, F. Radu, A. Tsukamoto, A. Itoh, A. Kirilyuk, T. Rasing, and A. Kimel, Phys. Rev. B 84, 024407 (2011). 
[25] E. Iacocca, T.-M. M. Liu, A. H. Reid, Z. Fu, S. Ruta, P. W. Granitzka, E. Jal, S. Bonetti, A. X. Gray, C. E. Graves, R. Kukreja, Z. Chen, D. J. Higley, T. Chase, L. Le Guyader, K. Hirsch, H. Ohldag, W. F. Schlotter, G. L. Dakovski, G. Coslovich et al., Nat. Commun. 10, 1756 (2019).

[26] U. Atxitia, J. Barker, R. W. Chantrell, and O. ChubykaloFesenko, Phys. Rev. B 89, 224421 (2014).

[27] J. Barker, U. Atxitia, T. A. Ostler, O. Hovorka, R. W. Chantrell, O. Chubykalo-Fesenko, and R. W. Chantrell, Sci. Rep. 3, 3262 (2013).

[28] J. Gorchon, C.-H. Lambert, Y. Yang, A. Pattabi, R. B. Wilson, S. Salahuddin, and J. Bokor, Appl. Phys. Lett. 111, 042401 (2017).

[29] U. Nowak, Handbook of Magnetism and Advanced Magnetic Materials (Wiley, Hoboken, NJ, 2007).

[30] U. Atxitia, O. Chubykalo-Fesenko, R. W. Chantrell, U. Nowak, and A. Rebei, Phys. Rev. Lett. 102, 057203 (2009).

[31] J. Nickolls, I. Buck, M. Garland, and K. Skadron, Queue 6, 40 (2008).

[32] M. I. Kaganov, I. M. Lifshitz, and L. V. Tanatarov, Zh. Eksp. Teor. Fiz. 31, 232 (1957) [JETP 4, 173 (1957)].

[33] J. K. Chen, D. Y. Tzou, and J. E. Beraun, Int. J. Heat Mass Transfer 49, 307 (2006).

[34] A. Ceballos, A. Pattabi, A. El-Ghazaly, S. Ruta, C. P. Simon, R. F. L. Evans, T. Ostler, R. W. Chantrell, E. Kennedy, M. Scott, J. Bokor, and F. Hellman, Phys. Rev. B 103, 024438 (2019).

[35] I. Radu, G. Woltersdorf, M. Kiessling, A. Melnikov, U. Bovensiepen, J.-U. Thiele, and C. H. Back, Phys. Rev. Lett. 102, 117201 (2009).
[36] A. Rebei and J. Hohlfeld, Phys. Rev. Lett. 97, 117601 (2006).

[37] B. Frietsch, J. Bowlan, R. Carley, M. Teichmann, S. Wienholdt, D. Hinzke, U. Nowak, K. Carva, P. M. Oppeneer, and M. Weinelt, Nat. Commun. 6, 8262 (2015).

[38] D.-H. Kim, T. Okuno, S. K. Kim, S.-H. Oh, T. Nishimura, Y. Hirata, Y. Futakawa, H. Yoshikawa, A. Tsukamoto, Y. Tserkovnyak, Y. Shiota, T. Moriyama, K.-J. Kim, K.J. Lee, and T. Ono, Phys. Rev. Lett. 122, 127203 (2019).

[39] C. E. Graves, A. H. Reid, T. Wang, B. Wu, S. de Jong, K. Vahaplar, I. Radu, D. P. Bernstein, M. Messerschmidt, L. Müller, R. Coffee, M. Bionta, S. W. Epp, R. Hartmann, N. Kimmel, G. Hauser, A. Hartmann, P. Holl, H. Gorke, J. H. Mentink et al., Nat. Mater. 12, 293 (2013).

[40] I. Radu, C. Stamm, A. Eschenlohr, F. Radu, R. Abrudan, K. Vahaplar, T. Kachel, N. Pontius, R. Mitzner, K. Holldack, A. Föhlisch, T. A. Ostler, J. H. Mentink, R. F. L. Evans, R. W. Chantrell, A. Tsukamoto, A. Itoh, A. Kirilyuk, A. V. Kimel, and T. Rasing, SPIN 5, 1550004 (2015).

[41] M. L. M. Lalieu, R. Lavrijsen, and B. Koopmans, Nat. Commun. 10, 110 (2019).

[42] R. F. L. Evans, T. a. Ostler, R. W. Chantrell, I. Radu, and T. Rasing, Appl. Phys. Lett. 104, 082410 (2014).

[43] C. Xu, T. A. Ostler, and R. W. Chantrell, Phys. Rev. B 93, 054302 (2016).

[44] C. Banerjee, N. Teichert, K. E. Siewierska, Z. Gercsi, G. Y. P. Atcheson, P. Stamenov, K. Rode, J. M. D. Coey, and J. Besbas, Nat. Commun. 11, 4444 (2020). 